\section{El enfoque epidemiologico del sistema de salud de Cuba}

\section{The epidemiologic approach of the Cuban health system}

\section{Silvia Martínez Calvo}

\section{Facultad Salud Pública}

Calle 146 No. 2504

e/ 31 y 25. Cubanacán, Playa.

Ciudad Habana. Cuba. CP 16

E-mail:ensap@ infomed.sld.cu

\section{Resumen}

El reconocimiento internacional a los logros del sistema de salud cubano, la estabilidad -en ocasiones cuestionada- de los indicadores de salud de la población, y los ingentes recursos humanos y materiales invertidos para desarrollar las políticas y estrategias sanitarias nacionales, constituyen un excelente ámbito para analizar la contribución de la Epidemiología a esos positivos resultados en el campo de la salud. Surgen dos provocadoras interrogantes: ¿Estuvo el sistema de salud epidemiológicamente orientado? $i$ Las acciones realizadas consienten la propuesta de una Escuela Cubana de Epidemiología? El propósito del trabajo es ofrecer respuestas a estas interrogantes através de una valoración crítica sobre la probable orientación epidemiológica del sistema y la incorporación o no del pensamiento epidemiológico en las estrategias sanitarias que se diseñan y desarrollan nacionalmente. Contribuye al análisis, una división arbitraria pero notoria: el Seminario sobre "Usos y perspectivas de la Epidemiología” realizado en Buenos Aires, que a partir de su fecha de realización en 1983, trazó pautas para el desempeño epidemiológico en la Región. Esa dicotomía - antes y después del Seminario- configuró los escenarios nacionales para la práctica epidemiológica en los niveles académico, investigativo y en los servicios, sostenidos en 4 pilares básicos: voluntad política, comunidad organizada y concientizada, sistema de salud con accesibilidad, cobertura total y financiamiento amplio, y por último, recursos humanos altamente calificados. El sistema de salud transita por etapas evolutivas cuyo bosquejo aporta elementos para reconocer su probable orientación epidemiológica, y su vinculación o no vinculación con las diferentes corrientes de pensamiento vigentes en cada etapa. Otro aspecto desafiante en el tema, es la propuesta de una Escuela Cubana de Epidemiología, y la valoración realizada en ese sentido, intenta mensurar la distancia entre lo ideal y lo real. En conclu- 
sión, se reconoce cierto vacío entre la argumentación teórica y la práctica epidemiológica en el Sistema Nacional de Salud, aunque sean inobjetables los avances en el ámbito sanitario en estas casi cuatro décadas. Resulta ineludible el desafío que esto representa para alcanzar el liderazgo epidemiológico que en el discurso se promueve, para lo cual la reforma del sector salud emerge como un excelente y oportuno espacio.

Palabras claves: Epidemiología. Sistema de salud. Estrategias nacionales. Recursos humanos en salud. Planes y programas de salud. Política de salud.

\section{Abstract}

The international recognition of the achievements of the Cuban health system, the stability-questioned on some occasions - of health indicators, and the enormous human resources and materials invested to develop the policies and national sanitary strategies comprise an excellent field to analyze the epidemiological contribution to those positive results in the health area. Among those outstanding results, the longer life expectancy, the low mortality rates, infant mortality, and mortality due to infectious and parasitic diseases partly due to the elimination or eradication of a group of diseases preventable by immunization. Were these results reached by an epidemiologically oriented health system? Do the procedures carried out allow for the proposal of a Cuban School of Epidemiology? The objective of this paper is to offer answers to these queries through a critical appraisal on the presumable epidemiological orientation of the health system, and the incorporation of epidemiological thinking in the sanitary strategies that have been designed and developed in Cuba. An arbitrary, but notorious dichotomy, the Seminar on the "Uses and Perspectives of Epidemiology", held in Buenos Aires in 1983, has traced rules for the epidemiological performance in the Region as a whole and contributed to the analysis above. The dichotomy -before and after the Seminar created the national scenarios for practicing epidemiology at the academic, investigative and health services levels, supported on 4 basic pillars: political will, an organized and conscious community, accessible health system, total coverage and broad financing, and highly qualified human resources. The health system went through four stages whose description - including healthcare models, development strategies, epidemiological activity and training - provides the means to recognize its presumably epidemiological orientation, and its link to the different schools of thought prevailing during each stage. Another important as- 
pect on the theme is the proposal of a $\mathrm{Cu}$ ban School of Epidemiology, and the assessment carried out, specifying the distance between the ideal thing and the real thing, because a "school of thought" does not seem "to be built by sum of well-known actions, if not, for overwhelming contributions that may re-formulate the discipline itself, and consequently reorient practice." In conclusion, a certain gap between the theoretic argument and the epidemiological practice in the National Health System is recognized. Consequently, its epidemiological orientation has been limited. The challenge the situation represents to reach the epidemiological leadership that is promoted in the discourse is unavoidable, and for this reason, health reform appears as an excellent and timely opportunity.

Keywords: Epidemiology. Health system. National strategies. Health manpower. Health planns and programmes. Health policy.
En el último lustro han existido en el ámbito académico nacional, algunas valoraciones sobre la orientación epidemiológica del sistema de salud, y la posibilidad de que esa práctica derivase en la ilusoria y efímera propuesta de una Escuela Cubana de Epìdemiología. Es criterio aceptado -al menos teóricamente- reconocer a la Epidemiología como la disciplina síntesis en la Salud Pública ${ }^{1}$, aunque no se perciba con nitidez su ubicación al interior de los sistemas de salud, y mucho menos se explicite su contribución en los resultados -positivos y negativos- de las políticas y estrategias sanitarias regionales en las últimas décadas. El tema resulta interesante, y el objetivo del trabajo no es solamente reconocer los logros y avances del sistema de salud cubano, si no ofrecer una opinión crítica sobre su probable orientación epidemiológica y la consecuente pretensión de una "escuela de epidemiología". Para desarrollar el análisis crítico se realiza un bosquejo del sistema nacional de salud en cuatro etapas evolutivas, desde su constitución en 1961 hasta la fecha, que incluye en cada una los aspectos siguientes: modelos asistenciales, estrategias de desarrollo, actividad epidemiológica y actividad formativa. Estimular los criterios, opiniones y valoraciones de los interesados en el tema, - que indudablemente serán asimilados - representa otro objetivo del trabajo.

En Cuba, los indicadores convencionales que miden la situación de salud de la población, muestran resultados positivos y estables durante los 35 años de desarrollo del sistema sanitario. Estos resultados estan vinculados básicamente a la intención política y la acción promocional del gobierno, y al desempeño de los cuantiosos recursos humanos que posee el sector salud y que según datos de 1998, para atender una población de 11,122,308 hts, se dispone de 339,943 trabajadores $-73,9 \%$ mujeres- , de los cuales $63483(57,81 \times 10,000 \mathrm{hts})$ son médicos, $9873(8,9 \times 10,000 \mathrm{hts})$ son estomatólogos y 82257 ( 74,2 x 10,000 hts) son enfermeras. Aunque suscribo el crite- 
rio de que el número de médicos no determina el estado de salud de una población, en este caso la orientación médico-centrista del sistema determina que la faceta asistencial recuperativa prevalezca, bien reconocida en una sociedad medicalizada y consecuentemente aceptada con beneplácito por la población.

Entre los indicadores que permiten evaluar el desarrollo -actualmente un tanto cuestionados- se destacan en la esfera socio-sanitaria la esperanza de vida y la tasa de mortalidad infantil. En el caso cubano, ésta última a partir de la implantación del programa de control en 1968, desciende paulatinamente de 38,7 por 1000 nacidos vivos en 1970 a 7,1 en 1998. Los recursos invertidos para el descenso son incalculables, tanto humanos como materiales, y la contribución epidemiológica se dirigió prioritariamente a disminuir la parte alícuota que en los inicios del programa aportaban a esas muertes las enfermedades transmisibles.En cuanto a la expectativa de vida, desde la década del 50 se observaba una tendencia a su elevación, que se confirma en décadas posteriores con la resultante de 74,2 años para los hombres y 78,0 para las mujeres, y una expectativa global de 76 años.

Un logro inobjetable de los programas de control epidemiológico fue el descenso de las tasas de mortalidad por enfermedades infecciosas y parasitarias, de 45,4 por 100,000 en 1970 a 9,8 por 100,000 en 1998 . Aunque el resultado de las actividades de control incluidas en los programas es sumamente positivo -siempre que estas se realicen con calidad- lo primordial en la orientación epidemiológica del sistema de salud no consiste en la administración de programas, en ocasiones de forma rutinaria, si no en la generación de conocimientos que se derivan de la utilización del instrumental científico de esa disciplina, diseminado en los diferentes niveles organizativos, y de la práctica consecuente en los servicios. Actualmente la situación epidemiológica se caracteriza por: acelerado envejecimiento, disminución de la natalidad y la fecundi- dad, incremento de la urbanización, deterioro de las condiciones higiénico ambientales, y como afecciòn más importante, las enfermedades crónicas no transmisibles ${ }^{2}$. En consonancia con la situación descrita y con el liderazgo que se atribuye a la Epidemiología en la Salud Pública, surgen algunas interrogantes: ¿Cómo contribuyó la Epidemiología a los resultados positivos? ¿Se diseminó y arraigó el enfoque epidemiológico al desarrollarse las estrategias sanitarias? ¿Los epidemiólogos aportaron ideas relevantes en el campo de la teoría y la práctica de su disciplina, o simplemente lo intentaron?.

Como el pensamiento epidemiológico ocupa una buena parte de este análisis, suscribo el enfoque conceptual expuesto por Nájera al respecto: "El pensamiento epidemiológico es aquel que pretende introducir el interés colectivo, el punto de vista comunitario o público en la respuesta que la sociedad ofrece a los problemas de salud, en una actitud de búsqueda e investigación permanente de las situaciones que generan o mantienen los problemas de salud" ${ }^{3}$.

Ni la eficacia de las acciones de promoción de salud desarrolladas directa e indirectamente por el gobierno, ni tampoco su reconocida contribución a la elevación del nivel de salud de los cubanos tuvieron una justa valoración ${ }^{4}$. El trabajo sectorizado de un organismo como el Ministerio de Salud Pública (MINSAP), responsabilizado con preservar y mantener la salud de la población contribuyó a cierto "olvido" de las consecuencias favorables de esa acción promocional, dirigida a modificar o eliminar factores negativos, que indudablemente influyen en el estado de salud de la población como son: el analfabetismo, la desnutrición, el desempleo, el ambiente físico agresivo, etc. La orientación preventiva de la estrategia sanitaria unida a la decisión política estimuladora y a las acciones promocionales gubernamentales, propiciaban el desarrollo de una percepción colectiva de salud que, a mi juicio, no fue cabalmente lograda.

El epidemiólogo cubano tuvo una situa- 
ción de privilegio, pues podía ejercitar sus habilidades técnicas apoyado en lo que propongo como los 4 grandes pilares del escenario nacional para la salud:

- voluntad política

- comunidad organizada y concientizada

- sistema de salud con accesibilidad total y financiamiento suficiente.

- recursos humanos altamente calificados con formación multidisciplinaria.

Estos elementos se convierten en requisitos básicos para alcanzar ese "pensar y vivir en salud" indispensable en la comunidad, y para simultáneamente establecer el liderazgo epidemiológico en el sistema de salud.

\section{Situación de la práctica epidemiológica antes del Seminario de Buenos Aires}

Para sustentar mejor el análisis, me atrevo a deslindar la situación del quehacer epidemiológico en Cuba en dos momentos: antes y después del Seminario de Buenos Aires en $1983^{5}$ que sobre Usos y Perspectivas de la Epidemiología reorientó el quehacer epidemiológico regional, aunque posteriormente surgieron nuevas valoraciones para mejorar ese quehacer, por ejemplo, el Informe Pemberton para la Comunidad Europea en 1986, la reunión de Taxco en $1987^{7}$, la reunión de Itapará, Brasil en $1988^{8}$ y la reunión de Ginebra en ese mismo año ${ }^{9}$ Estimo que el Seminario de Buenos Aires representó un hito importante para el desarrollo de la Epidemiología en la Región, y en el caso cubano, contribuyó a reafirmar en unos casos y a modificar en otros la práctica epidemiológica, que apoyada en el escenario que configuran los 4 pilares propuestos, se inicio al establecerse en 1961 el Sistema Nacional de Salud. Para ilustrar mejor ese accionar del sistema y la probabilidad de haberse orientado epidemiológicamente, en el cuadro se muestra un resumen de su evolución histórica, conformada por cuatro aspectos esenciales: modelo asistencial, estrategia de desarro- llo, actividad epidemiológica y actividad formativa.

Existe consenso nacional en reconocer que la evolución del sistema transcurre en cuatro etapas evolutivas, que coincdentemente acaecían cada 10 años. Se acompañaron de cambios decenales del modelo de prestación de servicios, aunque dadas las condiciones estatales del sistema de salud, en todas el modelo era de amplia cobertura, accesibilidad universal y con predominio de la asistencia integral priorizada para el nivel local. Simultáneamente los niveles de atención secundario y terciario alcanzaron gran desarrollo, y sobre todo, gran prestigio en la población que consecuentemente reforzó su preferencia por la faceta recuperativa.

Las estrategias de desarrollo en cada etapa promovieron espacios equivalentes al menos teóricamente- tanto para los servicios preventivos como para los curativos, aunque en la práctica se estimulaba el enfoque recuperativo, sustentado en el fortalecimiento y exaltación de los servicios hospitalarios y la promoción y divulgación de los éxitos de la atención médica. Es interesante como la población - aunque no todos - atribuya los grandes logros en la reducción masiva de enfermedades y daños casi exclusivamente al trabajo médico, e ignore el anónimo aporte que en ese sentido hicieron los profesionales y técnicos de las otras disciplinas de la Salud Pública. En cuanto a la actividad epidemiológica, estuvo centrada en los programas de control de enfermedades y daños, y en actividades de educación para la salud ejecutadas por el personal calificado ad hoc, pero con un enfoque eminentemente práctico. Por la importancia de esas actividades epidemiológicas para los objetivos del tema, merecen una valoración algo más amplia.

- Control y erradicación de enfermedades transmisibles. Esta actividad se desarrollaba mediante la estrategia de Campañas y Programas, que al decir de Loureiro, "estos programas contra daños respondían a la Epidemiología más cerca 


\begin{tabular}{|c|c|c|c|c|}
\hline Etapa & Modelo asistencial & Estrategia de desarrollo & Actividad Epidemiológica & Actividad Formativa \\
\hline I (1960-1970) & Medicina General & $\begin{array}{l}\text { - Implantación del Servicio } \\
\text { Médico Social Rural. } \\
\text { - Creación del Sistema } \\
\text { Nacional de Salud. } \\
\text { - Desarrollo de } \\
\text { infraestructura sanitaria: } \\
\text { policlínicos, hospitales, } \\
\text { asilos, clínicas } \\
\text { estomatológicas. } \\
\text { - Relaciones } \\
\text { intersectoriales para } \\
\text { actividades específicas. }\end{array}$ & $\begin{array}{l}\text { - Programas de control de } \\
\text { enfermedades y daños. } \\
\text { - Educación para la salud, } \\
\text { básicamente informativa. } \\
\text { - Participación comunitaria } \\
\text { en sus primeros grados. }\end{array}$ & $\begin{array}{l}\text { - Desarrollo de RH con } \\
\text { formación orientada al } \\
\text { "paradigma biológico." } \\
\text { - Traspaso de la } \\
\text { formación al sector } \\
\text { salud. }\end{array}$ \\
\hline II (1970-1980) & $\begin{array}{l}\text { Medicina en la } \\
\text { Comunidad }\end{array}$ & $\begin{array}{l}\text { - Creación del Policlínico } \\
\text { Comunitario. } \\
\text { - Transferencia } \\
\text { administrativa de la } \\
\text { infraestructura a los } \\
\text { gobiernos locales. } \\
\text { - Creación de los institutos } \\
\text { de investigación. }\end{array}$ & $\begin{array}{l}\text { - Mantenimiento de los } \\
\text { programas de control y } \\
\text { desarrollo de programas } \\
\text { de salud para la } \\
\text { protección integral de } \\
\text { grupos poblacionales. } \\
\text { - Participación comunitaria: } \\
\text { nivel de cooperación. } \\
\text { - Perfeccionamiento de la } \\
\text { educación para la salud. }\end{array}$ & $\begin{array}{l}\text { - Polos de formación en } \\
\text { la comunidad para } \\
\text { especialistas de: } \\
\text { medicina, pediatría y } \\
\text { gineco-obstetricia. } \\
\text { - Incremento en la } \\
\text { formación de técnicos } \\
\text { medios. }\end{array}$ \\
\hline III (1980-1990) & Medicina Familiar & $\begin{array}{l}\text { - Fortalecimiento red } \\
\text { servicios hospitalarios. } \\
\text { - Introducción y extensión } \\
\text { de altas tecnologías } \\
\text { extranjeras y nacionales. } \\
\text { - Creación de centros de } \\
\text { Hig.Y Epidemiologia } \\
\text { - Progr.de Atenc. Integral a } \\
\text { la Familia. }\end{array}$ & $\begin{array}{l}\text { - Diseño y ejecución del } \\
\text { programa nacional de } \\
\text { promoción. } \\
\text { - Modificaciones de las } \\
\text { técnicas educativas en la } \\
\text { comunidad. } \\
\text { - Reanimación de la } \\
\text { participación comunitaria } \\
\text { y la inspección sanitaria. } \\
\text { - Persistencia de } \\
\text { programas de control. }\end{array}$ & $\begin{array}{l}\text { - Creación de Facultades } \\
\text { de Ciencias Médicas en } \\
\text { todas las provincias. } \\
\text { - Desarrollo del } \\
\text { programa de } \\
\text { especialidades médicas. } \\
\text { - Creación de la Lic. de } \\
\text { Enfermería y Tecnología } \\
\text { de la Salud - Nuevo Plan } \\
\text { de Estudio de Medicina. }\end{array}$ \\
\hline IV (1990-2000) & Medicina Familiar & $\begin{array}{l}\text { - Perfeccionamiento de } \\
\text { APS. } \\
\text { - Participación social e } \\
\text { intrasectorial. } \\
\text { - Revitalización de } \\
\text { hospitales. } \\
\text { - Descentralización e } \\
\text { incremento del nivel } \\
\text { resolutivo del sistema. } \\
\text { - Desarrollo de la } \\
\text { tecnología de punta y la } \\
\text { investigación. } \\
\text { - Priorización de programas. }\end{array}$ & $\begin{array}{l}\text { - Análisis del sector salud. } \\
\text { - Desarrollo de estrategia } \\
\text { de riesgo. } \\
\text { - Creación de la red } \\
\text { Municipios por la salud. } \\
\text { - Desarrollo de la } \\
\text { farmacoepidemiología. } \\
\text { - Revitalización de } \\
\text { programas contra } \\
\text { enfermedades } \\
\text { reemergentes y } \\
\text { emergentes. }\end{array}$ & $\begin{array}{l}\text { - Capacitación a equipos } \\
\text { de dirección locales. } \\
\text { - Capacitación gerencial } \\
\text { a todos los niveles. } \\
\text { - Evaluación de la } \\
\text { competencia y el } \\
\text { desempeño } \\
\text { profesional. } \\
\text { - Perfeccionamiento del } \\
\text { programa de Medicina } \\
\text { Familiar. }\end{array}$ \\
\hline
\end{tabular}


de la Administración de Salud, y esto sucedía en la mayor parte de Latinoamérica desde los 70”0. En nuestro ámbito incluían actividades preventivas muy puntuales y respondían al principio del "carácter preventivo de la salud publica cubana"11. El "carácter preventivo" se desarrolló, pero no siempre en consonancia con las acciones promocionales de tipo gubernamental; era la epidemiología abordada desde su ángulo más tradicional, y - con excepciones - los epidemiólogos locales centraron su desempeño en las actividades de campo. Los resultados positivos de la aplicación de estas convencionales medidas de control, son inobjetables: eliminación de la poliomielitis, el paludismo autóctono, el tétanos del recién nacido, la difteria y otras prevenibles por vacunación, así como el control de las muertes por EDA en menores de 1 año, del dengue, etc. Estos programas contra enfermedades y daños aún permanecen como eje conductor del trabajo epidemiológico en los servicios, y en ese sentido la experiencia cubana puede generalizarse, independientemente del contexto específico de cada país o territorio.

- Educación para la salud. Dirigida esencialmente a ofrecer información sobre los principales problemas de salud y su prevención, tuvo resultados muy positivos al menos en las primeras décadas del sistema- al estimular la cooperación de la comunidad en la lucha contra enfermedades y daños. Las actividades educativas básicas eran esencialmente informativas como: charlas, audiencias sanitarias, debates de salud, mensajes y programas radiales, y en la última década se incoporan programas de TV. La comunidad a pesar de su organización y concientización no desarrolló espontáneamente toda su potencialidad, tal como reclama la orientación epidemiológica de un sistema de salud, aunque obviamente existió una valiosa cooperación en actividades sanitarias masivas. Resultó insólito como se eludieron los modelos de práctica social en salud vigentes en casi todas las sociedades contemporáneas, y aunque las actividades educativas contribuyeron a resolver trascendentes problemas de salud comunitaria -a pesar del marcado centrismo médico del sistema de salud -cabe preguntarse: ¿coincidían esas soluciones con las necesidades percibidas por la población?, ¿̇se observó con minuciosidad su práctica sanitaria?, ¿se indagó su comportamiento en ese sentido?.Si la población aún mantiene su avidez por la recuperación más que por vivir saludablemente, esa conducta revela la consecuencia de la direccionalidad de las estrategias en ese sentido al sustituir conscientemente la autorresponsabilidad individual, por el aparente beneficio colectivo.

Es notoria la actitud cambiante de la comunidad sobre su participación en la solución de problemas de salud, al transcurrir las diferentes etapas del sistema. En la primera y segunda etapas el nivel de compromiso social, de conciencia colectiva y la politización masiva, propiciaron su incorporación entusiasta y voluntaria a cualquier actividad relacionada con la salud; ya en la tercera etapa evolutiva el elevado nivel de información referente al tema, aumenta simultáneamente su nivel de exigencia hacia los servicios, que no su autorresponsabilidad por la salud, consecuencia directa de la autosuficiencia que también adquirió el propio sistema sanitario. En la etapa actual se ensayan nuevos enfoques para la participación comunitaria, que garanticen el logro de esta divisa de aquellos sistemas de salud que como el cubano, descansan en la Atención Primaria de Salud. Un ejemplo en ese sentido sería la actual Red de Municipios por la Salud. Todo este quehacer epidemiológico en los servicios debía acompañarse de un andamiaje teórico que lo justificara, hecho que no se percibió nitidamente a través del proceso evolutivo del sistema de salud. 
Desarrollo teórico de la Epidemiología. Según Buck, el pensamiento epidemiológico tiene limitaciones teóricas para la definición de políticas de salud ${ }^{12}$, y estimo que en el sistema nacional de salud esa situación fue evidente. La corriente de pensamiento que prevalecía en ese período era bastante ortodoxa, aunque matizada por influencias de algunos "centro emisores internacionales" como es el caso de la Oficina Sanitaria Panaméricana, cuyas Orientaciones Estratégicas y Programáticas se insertaban y apoyaban con sistematicidad. Por supuesto que estas "inserciones" y "apoyos" ajustados al pensar médico-clínico hegemónico prevelaciente y sin alterar los objetivos del sistema. Otra infuencia - aunque reducida - fue la "ideología sanitaria" representada por la Higiene Social, que proveniente del antiguo campo socialista, contribuyó a afianzar desde un enfoque "profiláctico", el control epidemiológico de los problemas de salud ${ }^{13}$.

Faltó modelo teórico, integración conceptual, o al menos un abordaje crítico, para demostrar convincentemente la contribución epidemiológica a los resultados, y en ello los epidemiólogos debemos asumir la responsabilidad. Se requiere de enfoques metodológicos sensatos, profundos, y sobre todo bien objetivos, para convencer de lo "novedoso y diferente" de nuestras acciones, si así fuese. Resulta difícil hacer aportes nuevos en cualquier ciencia por "joven” que sea - como es el caso de la epidemiología - y estimo que retomar categorías y principios científicos particulares, bien conocidos, y tratar de desarrollarlos en la práctica, no constituye en sí nada novedoso.

Un aporte sin objeciones, fue el desarrollo de procedimientos no convencionales en el abordaje del sujeto-objeto comunidad, como por ejemplo, el adiestramiento permanente de los líderes formales y no formales, las comisiones y grupos multidisciplinarios, las asambleas y audiencias sanitarias. Los aportes teóricos fueron aún más escasos y en ese sentido suscribo totalmente lo con- signado por Silva en un documento inédito: "padecemos de una considerable inhibición intelectual que nos cohibe de escribir, definir y someter al juicio externo nuestras ideas y concepciones $y$, en ese contexto (no fuera de él) nuestra experiencia. No existe tampoco un ejercicio sistemático de la crítica al trabajo teórico que se hace fuera de Cuba. Y, se da por añadidura, una tendencia a procurar reglas generales como sucedáneo del análisis y sus instrumentos naturales"*. La poca sistematicidad en la aplicación de corrientes epidemiológicas de punta no niega su conocimiento, ni en ocasiones su utilización correcta. Fue impostergable la necesidad de enfrentar los daños de mayor magnitud, pero ese "enfrentamiento" debía haberse acompañado de análisis reflexivos, de valoraciones sobre esa práctica epidemiológica que sin dudas la experiencia nacional enriqueció.

En cuanto a la inserción de pensares latinoamericanos dentro del quehacer epidemiológico cubano, desafortunadamente pareció existir una "invisible coraza" que impidió la aceptación de las corrientes de pensamiento epidemiológico-social desde sus "focos emisores" más connotados México, Brasil, Ecuador y Colombia. Los intentos de confrontación y análisis existieron aunque limitados a escasas instituciones, pues en las décadas del 70, 80 y hasta iniciados los 90, figuras emblemáticas de estas corrientes como Laurell, Breihl, Granda, Franco, Mercer, Castellanos, Loureiro, Eibenshutz, Betancourt, Nunes, entre otros, visitaron algunos centros docentes en Cuba, o asistieron a eventos nacionales. Aunque estos enfoques epidemiológico-sociales han sido merecedores de análisis fecundos concernientes a su validez metodológica y su aplicación en la práctica ${ }^{14}$, es innegable la audacia de la propuesta, con su enfrentamiento a los "poderosos positivistas norteños” y sus seguidores.

Eran válidas las expectativas de estos "grupos de pensamiento latinoamericano",

* Silva LC. Una reflexión sobre la consolidación de una Escuela Cubana de Epidemiología. Vicerrectoría de Investigaciones y Posgrado. Instituto Superior de Ciencias Médicas de la Habana. 1992 
acerca de la contribución del quehacer epidemiológico cubano a esos pensares, expectativas sustentadas en los presuntos vinculos teóricos entre los enfoques epidemiólogico-sociales y los procesos de transformación y estructura de una sociedad. Son inobjetables los cambios que originó el surgimiento del poder revolucionario en Cuba, pero los análisis de esas relaciones particulares entre la Epidemiología, los perfiles de salud y los cambios sociales, son hasta hoy, buenos propósitos. El desafío permanece "latente" y debe tener una respuesta asertiva.

Un excelente vínculo entre teoría y práctica debe darse en el ámbito académico, y al respecto su ejemplo más ilustrativo es la formación y superación de recursos humanos para la salud que representó un importantísimo elemento en el desarrollo del sistema de salud en Cuba. Según los datos del cuadro, la formación parece estar en consonancia -en todas las etapascon la estrategia de desarrollo prevaleciente, que teóricamente se apoyaba en acciones promocionales y preventivas, aunque en el paradigma formativo predominara un enfoque biológico. Independientemente de las buenas intenciones académicas de la última década, estimo que aún transcurrirá tiempo para que el médico de familia se aproxime a la comunidad desde su ambiciosa y promisoria función de “promotor y preventólogo", pues hasta hoy esa intención está frecuentemente vinculada con la atención médico-clínica, traducida en la visita a enfermos ó a individuos en riesgo.

La formación epidemiológica reprodujo los modelos formativos más socorridos, que priorizaban los enfoques descriptivos del problema y la ejecución de medidas elementales de control, y resulta paradójico que una parte considerable de los profesores cubanos no se formaron ni en EUA, ni en Inglaterra, y sin embargo, el modelo formativo reproducido hasta la saciedad, es casi una copia de esos patrones, aunque sin menoscabo de los aportes "criollos".

El problema esencial de la capacitación epidemiológica radicó en lo convencional del proceso formativo y las limitaciones para un desempeño consecuente ${ }^{15}$. Los curriculums -con excepciones- mantuvieron el abordaje maniqueísta de la clásica división representada por las enfermedades transmisibles y las enfermedades crónicas no transmisibles. Ese modelo formativo afortunadamente superado - propició que generaciones de epidemiólogos no desarrollaran su cualidad predictiva en el desempeño y no percibieran el "agotamiento" del modelo de atención médica en la década del 80. El interés y la pertinacia política si lo percibió y surgió la propuesta oportuna del nuevo modelo medico y enfermera de la familia ${ }^{16}$; fue este un momento crucial para desarrollar la orientación epidemiológica que se intenta desde los inicios del Sistema Nacional de Salud.

Esas "debilidades" formativas con certeza contribuyeron a entorpecer la orientación epidemiológica del sistema, pero no impidieron totalmente ciertos avances de la Epidemiología como disciplina. En ese sentido considero injustas las críticas emitidas por prestigiosos epidemiólogos, y que desafortunadamente encontraron eco en el país, con olvido del patrón de comparación utilizado. Recuerdo una conversación muy interesante con el prof. Milton Terris hace unos años, en que reiteraba su criterio -emitido en una conferencia previa- sobre la carencia en Cuba de investigaciones observacionales rigurosas, al estilo de los grandes centros de investigación y universidades de Norteamérica y Europa. Es pertinente recordar que mientras en los países desarrollados los paladines de la Epidemiologia "dura" avanzaban, los epidemiólogos cubanos enfrentaban difíciles problemas de salud heredados del subdesarrollo sanitario, e instrumentalizaron una práctica epidemiológica coherente, aunque convencional, con la necesidad de transformar ese desfavorable perfil. Simultáneamente asumían la formación masiva de recursos humanos para contribuir al control o eliminación de esos graves problemas sanitarios. A pesar de su tradicionalismo científico no 
deben abandonarse estas actividades permanentes de control consideradas rutinarias, y menos aún desmantelar la infraestructura preventivo-assistencial desarrollada como respuesta a grandes daños a la salud léase enfermedades de todo tipo en particular las transmisibles- cuya utilidad es innegable y más con la amenazante situación epidemiológica que representa la emergencia y reemergencia de enfermedades transmisibles. Como bien consigna el propio M.Terris "la epidemiología es una ciencia de campo, cualquier intento de separarla de la realidad concreta para transformarla en algo puramente académico, es destruirla ${ }^{17}$. Reconozco que el sistema ofrece ya espacios para reflexionar sobre las ventajas y desventajas de la exclusiva prioridad de los programas de control en los servicios, y que las condiciones son propicias para el avance científico-técnico de la Epidemiología en el país, de forma integral y abarcadora que desarrolle sus usos principales.

Aunque "se requieren profesionales más capaces con potencial para el análisis, interpretación, creación y aplicación de los nuevos conocimientos apropiados para la complejidad de los problemas actuales" estimo que un "curso de epidemiología" no transforma vertiginosamente un pensamiento médico hegemónico en un pensamiento epidemiológico. Existe plena convicción en los decisores de que es impostergable el re-adiestramiento técnico-metodológico de los recursos humanos para enfrentar los nuevos desafíos epidemiológicos, y que esta situación actualmente constituye una urgencia para el sistema de salud.

Finalmente merece reconocimiento en esta etapa el aporte de cientos de epidemiólogos, higienistas, administradores de salud, enfermeras, ingenieros sanitarios, microbiólogos, químicos, técnicos de Higiene y Epidemiología, psicólogos, trabajadoras sociales, etc, que desde el campo de la prevención, participaron activamente para lograr avances, y que también compartieron las insuficiencias y limitaciones de esa práctica.

\section{Situación de la práctica epidemiológica después del Seminario de Buenos Aires}

¿Cómo se recibieron, adaptaron y aplicaron las propuestas regionales en el ámbito sanitario cubano?. Considero que las propuestas regionales que dimanaron del Seminario, básicamente resumen y redimensionan los clásicos usos de la disciplina ${ }^{19}$, y esencialmente se concentraron en:

- Análisis de la situación de salud

- Desarrollo de investigaciones causales

- Vigilancia epidemiológica

- Evaluación de programas, servicios y tecnología.

Antes del Seminario, estos “usos” tenían aplicación en el Sistema de Salud -unos con más prioridad que otros - y algunos distantes del enfoque epidemiológico requerido, dígase la evaluación de programas, servicios y tecnología, totalmente orientada a sus aspectos administrativos y con énfasis en el cumplimiento de metas; representaba un modelo en desarrollo de lo que Castellanos considera como "limitaciones para una mejor articulación de la Epidemiología con la planificación, organización y gestión de los servicios de salud"20.

Como resultado del Taller OPS/OMS realizado en Cuba, y en consonancia con la implantación del nuevo modelo de Medicina Familiar, el área de Higiene y Epidemiología del Ministerio de Salud Pública (MINSAP), patrocinó 2 importantes y promisorios Seminarios-Talleres en $1987 \mathrm{y}$ $1988^{21}$. Este modelo de Medicina Familiar se implantó nacionalmente en 1984 y se convirtió en la "piedra angular" de la estrategia sanitaria; su aparición originó nuevas necesidades de adiestramiento y re-calificación de profesionales y técnicos, básicamente en el campo de la Epidemiología. Los resultados de los "debates masivos" en ambos Talleres, se tradujeron en orientaciones concretas para revitalizar el quehacer epidemiológico -al menos teóricamentedentro del sistema nacional de salud. La consecuencia más relevante fue el diseño y 
ejecución en los inicios de la década de los 90 de la estrategia sanitaria reconocida nacionalmente por sus siglas OPD/2000 (Objetivos, Propósitos y Directrices) no dependiente en su totalidad de resultados de investigaciones epidemiológicas rigurosas, pero estimo que esa estrategia representó uno de los más intrépidos propósitos de orientar epidemiológicamente el sistema nacional de salud ${ }^{22}$. La estrategia descansaba casi completamente en el enfoque de riesgo, tanto individual como poblacional, y su implementación se acompañó de un Proyecto de Capacitación para facilitar su su desarrollo por parte de los equipos locales de salud. Este proyecto se ejecutó nacionalmente durante 5 años (1992-1997), con un enfoque metodológico que a juicio de expertos, resultó ágil y motivador. Se diseñó y ejecutó con la cooperación técnica de OPS/OMS y fue divulgado en las Facultades y Escuelas de Salud Pública de la Región ${ }^{23}$.

Actualmente se desarrolla en el país una estrategia sanitaria más "puntual”, que está resumida en una Carpeta Metodológica que facilita su ejecución y evaluación, y que impulsa también acciones promocionales mediante redes de Municipios por la Salud - "La Carpeta Metodológica establece la doctrina de trabajo del sector y sus procedimientos, favoreciendo la integración de acciones en la misma dirección para garantizar el cumplimiento de las Estrategias y Programas Priorizados. Implantada en 1996, es objeto de un proceso de revisión y enriquecimiento continuo. Esta flexibilidad $y$ adaptabilidad a las condiciones existentes, la hacen un instrumento de gran valor para la normalización y estandarización de los procesos técnicos. Se han elaborado Carpetas Metodológicas en las áreas técnicas, ingenieras, administrativas, económico-financieras y de servicios ${ }^{24}$.

En este contexto post-seminario, se desarrolló aún más la red de vigilancia epidemiológica - el más común y en nuestro caso el más diseminado de los usoscuyo soporte era el eficiente subsistema de información de estadísticas sanitarias, que abarca hasta los niveles locales en todo el país; una limitante era la excesiva centralización de las actividades de análisis e interpretación de datos, y además orientadas casi exclusivamente a las enfermedades transmisibles ${ }^{25}$. Los avances tecnológicos renovaron aquella red inicial de vigilancia epidemiológica, y ésta se incluyó en el recién implantado sistema de Vigilancia en Salud a través de una estructura sólida y verticalizada, y un equipamiento tecnológico bien moderno. Se amplió el espectro al incluir otros "objetos a vigilar": como: agresiones ambientales, índice ocupacional en hospitales, disponibilidad de medicamentos, etc ${ }^{26}$.

El Análisis de la situación de salud, que según Castellanos "tuvo poca repercusión en los servicios en la mayoría de los países $^{27}$, acompaña al sistema nacional de salud desde sus inicios, aunque ha transitado por diferentes momentos en su utilización. Acuñado como diagnóstico de salud su uso no se sistematiza en los servicios y en la década del 70 renace al desarrollarse un nuevo modelo de atención denominado Medicina en la Comunidad, e incluirse como elemento práctico en las especialidades básicas de la Atención Primaria ${ }^{28}$. Sin dudas su "refugio privilegiado" fue el ámbito académico, y con el surgimiento del modelo de Medicina Familiar en la década del 80 , resurge el análisis de la situación de salud como instrumento técnico-metodológico idóneo y como habilidad singular del Médico de Familia, que durante los 3 años de su etapa de residente realiza análisis semestrales en la comunidad que atiende ${ }^{29}$.

La investigación causal se circuncribe básicamente a los centros nacionales de investigación biotecnológica, clínica, ambiental y ocupacional, aunque en los centros docentes también se desarrollen investigaciones -prioritariamente descriptivasvinculadas al proceso formativo y concernientes a problemas de salud importantes para el ámbito local. Merecen elogios las experiencias positivas de los profesionales ubicados en los Institutos de investigación, 
que desde su especialización básica, por ejemplo, cardiología, endocrinología, neurología, oncología, nutrición, etc, desarrollan investigaciones epidemiológicas observacionales multicéntricas, tanto dentro como fuera del país. Los avances son obvios en cuanto a investigación biológica, genética e inmunológica se refiere; la producción de vacunas inducida por la trascendencia y magnitud de algunas enfermedades transmisibles -es emblemático lo ocurrido con la enfermedad meningocóccicaha servido de puente entre la investigación epidemiológica observacional, los ensayos de campo y esa producción biológica.

Los aportes epidemiológicos "puros" derivados de las investigaciones son infrecuentes, pues lo común-con excepciones- es reproducir esquemas investigativos "probados" internacionalmente y ajustados al ámbito nacional. Una excepción en este sentido fue la relevante participación nacional en dos líneas de investigación propuestas por OPS/OMS en la década del 80, y dentro del Programa Análisis de situación de salud y sus tendencias: Diferenciales de mortalidad y Vigilancia de la situación de salud según condiciones de vida, cuyos resultados fueron presentados en varios Boletines Epidemiológicos de ese período ${ }^{30,31}$. Son escasas las denominadas investigaciones comunitarias, así como los estudios ecológicos, confundidos con estudios descriptivos sobre problemas comunitarios. Finalmente parecen resurgir con "cuerpo propio" las investigaciones en sistemas y servicios de salud al reclamo de la actual estrategia sanitaria que intenta mantener y superar los indicadores de salud, y también como respuesta a las orientaciones de las políticas regionales en ese campo ${ }^{32}$.

Deliberadamente dejo en último lugar lo ocurrido con la propuesta para evaluación de programas, servicios y tecnologías, pues ha sido, a mi juicio, la actividad más endeble del sistema de salud, al menos desde su enfoque epidemiológico. Las evaluaciones "administrativas" prevalecen en afán de medir eficacia y eficencia, y con preferencia eluden las valoraciones del impacto epidemiológico de programas y servicios. Lo ocurrido en Cuba es reflejo externo, pues este tipo de actividad "evaluativa" es también reducida en la mayoría de los países en desarrollo; actualmente se reivindica su validez epidemiológica para los servicios, estimulada-entre otros elementos- por los modelos que preconizan las instituciones formadoras. Esporádicamente surge alguna modalidad evaluativa ante "urgencias epidemiológicas”, pero no representa una acción sistemática incorporada al quehacer de los servicios de salud. Alcanza vigencia la evaluación de tecnología, desde una estructura organizativa que parece promisoria, pero estimo que aún son intentos encomiables con resultados inciertos y a largo plazo. Regionalmente se sienten "nuevos aires" para este importante uso de la Epidemiología, el más relegado en su aplicación, y uno de los más relevantes y sugerentes en cuanto a resultados.

\section{Comentarios finales}

Con análisis crítico desde dos escenarios conocidos intente ofrecer respuestas a las interrogantes sobre la existencia o no, de la orientación epidemiológica del Sistema Nacional de Salud cubano y de una Escuela Cubana de Epidemiología. Los criterios derivan de la experiencia personal en funciones técnico administrativas en diferentes niveles organizativos del sub-sistema de Higiene y Epidemiología, y como consigné, la carencia de una masa crítica nacional constituída por los epidemiólogos comprometidos con los resultados alcanzados, redujo las posibilidades de un debate interno sobre el tema, aunque existieron intentos fallidos en los finales de la década del 80 y también recientemente. Por consiguiente, entre mis objetivos está promover opiniones y comentarios de otros colegas que, dentro o fuera de la región, se han interesado en "descubrir" las bases teóricas que regulan los avances del sistema de salud cubano, y no exclusivamente en los procedimientos para lograrlo.

Más que orientación epidemiológica del 
Sistema Nacional de Salud, afirmo que se desarrolló una aplicación audaz del enfoque epidemiológico más tradicional, acompañando la hegemónica direccionalidad médico-centrista prevaleciente. La erradicación, eliminación y control de enfermedades transmisibles -que en ocasiones representaban una amenaza y en otras una adversa realidad- no debe estimarse como resultante absoluta del "liderazgo epidemiológico", si no un resultado efectivo de la conjunción coyuntural entre los 4 pilares básicos que se requieren para el trabajo epidemiológico dentro del sistema de salud. Por otro lado, la efímera propuesta de una Escuela Cubana de Epidemiología es también intrépida pues una escuela de pensamiento no parece "construirse" con la suma de acciones conocidas, si no por contundentes aportes que contribuyan a enaltecer y reformular la propia disciplina y a reorientar su práctica, tal como verdaderamente sucedió con la conformación de la Escuela Cubana de Pediatría a partir de $1920^{33}$. El loable propósito de que exista una escuela de Epidemiología en nuestro ámbito, no significa que se haya logrado.

Desde los servicios no deben ofrecerse respuestas sobre la vinculación de los argumentos teóricos con la práctica epidemiológica cotidiana, restaría entonces al campo investigativo y al académico reivindicar esa teorización imprescindible, como confirmación de los avances que obviamente ha logrado el sistema de salud. Suscribo el planteamiento de que "el marco social de cada país constituye un condicionante del grado de desarrollo de la comunidad científica y del grado de fortaleza y legitimidad de sus instituciones, así como del nivel de valoración del conocimiento científico-técnico como fundamento de las decisiones" ${ }^{\prime 3}$, y al mismo tiempo considero atinado y oportuno aprovechar la actual coyuntura de reorientación del sistema dentro de las estrategias de reforma del sector salud, para desarrollar el liderazgo epidemiológico que en el discurso se promueve.

\section{Summary}

The international recognition of the achievements of the Cuban health system, the stability - questioned on some occasions - of health indicators, and the enormous human resources and materials invested to develop policies and national sanitary strategies comprise an excellent field to analyze the epidemiological contribution to those positive results in the health area. Among the outstanding results, longer life expectancy, low mortality rates, infant mortality, and mortality due to infectious and parasitic diseases, partly due to the elimination or eradication of a large group of diseases preventable by immunization. Was the health system epidemiologically oriented? Do the procedures carried out allow for the proposal of a Cuban School of Epidemiology? The objective of this paper is to offer answers to these queries through a critical appraisal on the presumable epidemiological orientation of the health system, and the incorporation of epidemiological thinking in the sanitary strategies designed and developed in Cuba. The Seminar on the "Uses and Perspectives of Epidemiology" was an arbitrary but notorious dichotomy. Since 1983, when it was held in Buenos Aires, it has outlined rules for the epidemiological performance in the Region as a whole, and has contributed to the analysis above. The dichotomy -before and after the Seminar created the national scenarios for practicing epidemiology at the academic, investigative and health service levels, supported on 4 basic pillars: political will, an organized and conscious community, an accessible health system, total coverage and broad financing, and highly qualified human resources. The health system went through stages whose description - including healthcare models, development strategies, epidemiological activity and training - provides the means to recognize its presumably 
epidemiological orientation, and its link to the different schools of thought prevailing during each stage. The control and eradication of communicable diseases, health education activities, and education and training of human resources, reveal the interest in developing the classic inherent promotional and preventive interventions for the health system as a whole. Sanitary actions are heaped, and the "good intentions" are not linked with an advanced theoretic position that justifies the epidemiological work. Another important aspect of the theme is the proposal of a Cuban School of Epidemiology, and the assessment carried out, specifying the distance between what is ideal and the real thing, because a school of thought does not seem "to be built by the sum of well-known actions, if not, by overwhelming contributions that may reformulate the discipline itself, and consequently reorient the practice." In conclusion, a certain gap between the theoretic argument and the epidemiological practice in the National Health System is recognized. Consequently, its epidemiological orientation has been limited. The advances in the sanitary field in these almost four decades may be derived from the fact that services use the most traditional epidemiological procedures, directly linked to what was considered as the basic pillars that sustain the scenarios for the epidemiological work. The technical-methodological applications that have been developed in a pointed and vertical form contribute very little to sustain the actions on rigorous analysis, on integral proposals, and rarely to focus on the inherent predictable features recognized in the epidemiological orientation of the health system. The challenge this situation represents to reach the epidemiological leadership that is promoted in the discourse is unavoidable, and for this reason, health reform appears as an excellent and timely opportunity.

\section{Referencias}

1. Macedo CG. Discurso apertura. In: Documento del Seminario sobre "Usos y Perspectivas de la Epidemiología”; 1983 Nov 7-10; Buenos Aires, Argentina. (Publicación No. PNSP 84-47/1984)

2. Hadad J. El sistema nacional de salud: evolución, evaluación, estrategias y estilos. In: Fernández E, Castell Florit P, compiladores. Gerencia en salud. São Paulo: Facultad Salud Pública; 1998. p. 190.

3. Nájera E. Investigación y desarrollo profesional. In: Oficina Sanitaria Panamericana. La formación en epidemiología para el desarrollo de los servicios de salud. Washington (DC); 1987. (Serie Desarrollo de Recursos Humanos, 88).

4. Martínez S. La promoción de la salud en la estrategia sanitaria cubana. La Habana; 1994. (Publicacion Grupo de Estudios Interdisciplinarios en Salud).

5. Seminario sobre "Usos y Perspectivas de la Epidemiología”; 1983; Buenos Aires, Argentina. (Publicación No. PNSP 84-87/1984).

6. Organización Panamericana de la Salud. Fortalecimiento de la práctica de la epidemiología en la comunidad europea. Bol Epidemiol 1988; 8: 10-4.

7. Organización Panamericana de la Salud. La formación en epidemiología para el desarrollo de los servicios de salud. Washington (DC); 1989. (OPS - Serie Desarrollo de Recursos Humanos, 88).

8. Organización Panamericana de la Salud. Diagnóstico de la situación de salud en Brasil: Conclusiones del Seminario sobre las Perspectivas de la Epidemiología frente a la Reorganización de los Servicios de Salud. Bol Epidemiol 1987; 8: 6-9.

9. Organización Panamericana de la Salud. Usos de la epidemiología en apoyo de las estrategias de salud para todos. Bol Epidemiol 1989; 10: 15.

10. Loureiro S. La formación académica en epidemiología. In: Organización Panamericana de la Salud. La formación en epidemiología para el desarrollo de los servicios de salud. Washington (DC); 1989. (OPS -Serie Desarrollo de Recursos Humanos, 88).

11. Hernández R. Administración de salud. La Habana: Editorial Ciencia y Técnica/Instituto Cubano del Libro; 1971.

12. Buck C. Epidemiología y política de salud. In: Organización Panamericana de la Salud. La formación en epidemiología para el desarrollo de los servicios de salud. Washington (DC); 1987. (OPS-Serie Desarrollo de Recursos Humanos, 88). 
13. Serenko AF, Ermákov VV. Fundamentos de la higiene social. La Habana: Instituto de Desarrollo de la Salud; 1985.

14. Almeida N. La investigación y la práctica de la epidemiología en América Latina: desafíos teóricos y metodológicos. p. 1-10. [Documento de trabajo para el Taller "Situación de salud segun condiciones de vida"; 1993; Salvador, Bahía].

15. Caraballoso-Hernandez M, Granda-Ibarra A, CabreraTrujillo JF, del Puerto-Guintana C, Hernandez Ceballos $\mathrm{H}$, Martinez-Calvo S et al. Aspectos generales del Programa Nacional de Formación del Especialista en Higiene y Epidemiología de Cuba. Educ Med Salud 1990; 24: $280-94$.

16. Cuba. Ministério de Salud Publica. Programa de Trabajo del Médico y Enfermera de la Familia, el Policlínico y el Hospital. La Habana; 1988.

17. Boyer M, Ferreira JR. Iniciativa ALAESP/ASPH/OPS para el liderazgo en Salud Pública. In: Organización Panamericana de la Salud. La formación en epidemiología para el desarrollo de los servicios de salud. Washington (DC); 1987. (OPS-Serie Desarrollo de Recursos Humanos, 88).

18. Terris M. La tradición epidemiológica. In: La revolución epidemiológica y la medicina social. $2^{\mathrm{a}}$ ed. México (DF): Editorial Siglo XXI; 1982. p. 24. (Colección "Sociedad y Salud").

19. Morris J. The uses of epidemiology. Edinburgh: Churchill Livingstone; 1957.

20. Castellanos PL. Epidemiología y organización de los servicios. In: Organización Panamericana de la Salud. La formación en epidemiología para el desarrollo de los servicios de salud. Washington (DC); 1987. (OPS-Serie Desarrollo de Recursos Humanos, 88).

21. Organización Panamericana de la Salud. Taller Nacional de Epidemiología en los Servicios de Salud y el Médico de la Familia en Cuba. Bol Epidemiol 1988; 9: 11-5.

22. Cuba. Ministério de Salud Pública. Objetivos, propósitos y directrices para incrementar la salud de la población cubana. 1992-2000. La Habana; 1992. [Documento de trabajo].
23. Martínez S. Proyecto de superación a equipos de dirección del sistema nacional de salud. La Habana; Ministerio de Salud Pública; 1995.

24. Propósitos del sistema nacional de salud para el período 1996-2000. In: Fernández E, Castell-Florit P, compiladores. Gerencia en salud . La Habana: Facultad Publica de Salud; 1998. p. 190.

25. González E. Sistemas de vigilancia epidemiológica. La Habana: Editorial Ciencias Médicas; 1989.

26. Cuba. Ministerio de Salud Pública. Sistemas de vigilancia en salud. La Habana; 1996. [Documento sobre el desarrollo y perspectivas de la Vigilancia en Salud en Cuba. Unidad de Análisis y Tendencias en Salud].

27. Organización Panamericana de la Salud. Seminario sobre Usos y Perspectivas de la Epidemiología; 1983 Nov 7-10; Buenos Aires, Argentina. Washington (DC): 1984. (OPS-PNSP 84-47).

28. Ordoñez C, Casals A. La participación comunitaria en el nuevo modelo de Atención Primaria de Salud. Rev Cubana Adm Salud 1978; 4:63-9.

29. Cuba. Ministerio de Salud Publica. Programa de especialización en Medicina General Integral. La Habana: Edición Provisional; 1990. v. 1.

30. Organización Panamericana de la Salud. Mortalidad en las Américas 1950-1990. Bol Epidemiol 1992; 13: 4-8.

31. Organización Panamericana de la Salud. Diferenciales de salud según condiciones de vida. Líneas futuras de investigaciones sobre perfiles de salud. Bol Epidemiol 1988; 9: 1 .

32. Organización Panamericana de la Salud. Orientaciones estratégicas y programáticas 1995-1998. Washington (DC); 1995. (OPS-Documento Oficial, 269).

33. López Serrano E. Aspectos de la historia de la pediatría en Cuba. El 60 aniversario de la Fundación de la Sociedad y de la Revista Cubana de Pediatría. Rev Cubana Pediatr 1988; 60: 797-803.

34. Tigre CH, Plaute R, Libel M, Castellanos PL, BrandlingBennett D, Arnt N et al. La práctica epidemiológica en los sistemas de servicios de salud. Educ Med Salud 1990; 24; 306-20. 